\title{
NUMERICAL HOMOGENIZATION OF MONOTONE ELLIPTIC OPERATORS*
}

\author{
YALCHIN EFENDIEV ${ }^{\dagger}$ AND ALEXANDER PANKOV
}

\begin{abstract}
In this paper we construct a numerical homogenization technique for nonlinear elliptic equations. In particular, we are interested in when the elliptic flux depends on the gradient of the solution in a nonlinear fashion which makes the numerical homogenization procedure nontrivial. The convergence of the numerical procedure is presented for the general case using $G$-convergence theory. To calculate the fine scale oscillations of the solutions we propose a stochastic two-scale corrector where one of the scales is a numerical scale and the other is a physical scale. The analysis of the convergence of two-scale correctors is performed under the assumption that the elliptic flux is strictly stationary with respect to spatial variables. The nonlinear multiscale finite element method has been proposed and analyzed.
\end{abstract}

Key words. homogenization, multiscale, finite element, random, monotone

AMS subject classification. 65N99

DOI. $10.1137 / \mathrm{S} 1540345903421611$

1. Introduction. Multiscale problems occur in many scientific and engineering disciplines, such as material science, earth and environmental science, petroleum engineering, etc. These problems are characterized by the great number of spatial and time scales. They are difficult to analyze theoretically or solve numerically.

When a standard finite element or finite difference method is applied to the multiscale problems, the degrees of freedom of the resulting discrete system can be extremely large due to the necessary resolution for achieving meaningful (convergent) results. Limited by computing resources, many practical problems are still beyond the reach of direct simulations. On the other hand, the large-scale features of the solutions are often of main interest. Thus, it is desirable to have a numerical method that can capture the effect of small scales on large scales without resolving the small-scale details.

In this paper we consider nonlinear elliptic equations where the flux depends on the gradient of the solutions in a nonlinear manner:

$$
-\operatorname{div}\left(\mathbf{a}_{\epsilon}\left(x, D u_{\epsilon}\right)\right)=f,
$$

where $\epsilon$ is a small scale. In the previous findings, MsFEM (see $[10,9,7,8]$ ) and other upscaling techniques (see, e.g., $[21,5,2]$ ) have been successfully applied to linear elliptic equations. In this case the coarse scale solution can be constructed by rescaling of the local solution or the coupling of the local solution through the variational formulation of the problem. This technique is no longer applicable for nonlinear elliptic equations when the elliptic flux depends on the gradient of the solution in a nonlinear fashion.

Our motivation in considering (1) mostly stems from the applications of flow in porous media, though many applications of nonlinear elliptic equations of these

${ }^{*}$ Received by the editors January 24, 2003; accepted for publication (in revised form) September 3, 2003; published electronically November 25, 2003.

http://www.siam.org/journals/mms/2-1/42161.html

†Department of Mathematics, Texas A\&M University, College Station, TX 77843-3368 (efendiev@ math.tamu.edu).

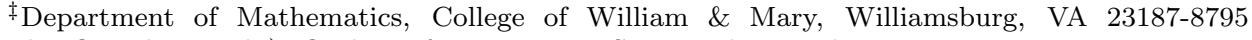
(pankov@math.wm.edu). On leave from Vinnitsa State Pedagogical University. 
kinds occur in transport problems. Two-phase immiscible flow in porous media is governed by the pressure equation $\nabla \cdot \lambda(S) \mathbf{k} \nabla p=f$, and the saturation equation $S_{t}+\mathbf{v} \cdot \nabla f(S)=0$, where $\mathbf{v}=-\lambda(S) \mathbf{k} \nabla p$. The pressure field, $p$, is the solution of the nonlinear elliptic equation, where the elliptic flux depends on the saturation $S$ through the function $\lambda(S)$. On the other hand, $S$ depends on the velocity field $\mathbf{v}$, which in turn is a function of $\nabla p$. Taking the present value of the velocity in the saturation equation yields the form (1) for the pressure equation. A simple way to view it is to solve the saturation equation explicitly with the pressure implicit, i.e., $S(\cdot, t)=S(\cdot, t-\Delta t)+\Delta t \lambda(S(\cdot, t-\Delta t) k(\cdot) \nabla p(\cdot, t) \nabla f(S(\cdot, t-\Delta t)$, and substitute this value of the saturation into the pressure equation. The resulting pressure equation will contain the gradient of the pressure in a nonlinear fashion.

The paper deals with two main issues: (1) the calculation of the homogenized solution on a numerical coarse grid and (2) the computation of numerical correctors that approximates the gradient of the solution. It is known that the solution $u_{\epsilon}$ converges to $u$ (up to a subsequence), which is the solution of $-\operatorname{div}\left(\mathbf{a}^{*}(x, D u)\right)=f$ (e.g., see [16, p. 90]). In the periodic case the solution $u_{\epsilon}$ has the following multiscale expansion

$$
u_{\epsilon}=u_{0}+\epsilon u_{1}(x, x / \epsilon)+\cdots,
$$

where $u_{1}$ can be expressed through $u_{0}$ (see, e.g., [1]).

In the first part of the paper we propose a numerical procedure for the calculation of the homogenized solution at length scales $h, 1 \gg h \gg \epsilon$. Our numerical homogenization procedure is based on the general finite element formulation of the coarse scale equations. It selectively solves the local problems that reduce overall computations even in the periodic case. In this part of the paper we also prove the convergence of the numerical solution computed on the coarse grid to the solution of the homogenized equation. The proof is presented for the general case using $G$ convergence theory. We show that the discrete problem has a unique solution, and this solution converges (up to a subsequence) to the solution of the discretized problem for a homogenized equation. Next, the nonlinear multiscale finite element method is presented, and the similarity with the proposed numerical homogenization technique is discussed. The nonlinear multiscale finite element is a Petrov-Galerkin finite element method where the "test space" and trial space are different. In particular, the trial space is chosen to be the space of usual linear bases, while the "test space" is a manifold (not a linear space) that can be mapped from the trial space in a nonlinear manner.

The second part of the paper deals with the construction of the fine scale features of the solution. One of our goals here is to build a numerical corrector. To the best of our knowledge, there is no corrector result for the random case. In the paper we construct the two-scale correctors for the random operators that are strictly stationary with respect to spatial variables. Our approach borrows some ideas from the periodic case [4]. A distinctive feature of our approach is the introduction of stochastic twoscale correctors, where one of the scales represents the numerical (coarse) grid size and the other scale is a physical scale. The convergence for the corrector is obtained. These results show us a way to obtain numerically the fine scale features of the solution. Moreover, the nonlinear multiscale finite element method as in the linear case recovers the fine scale features of the solution gradient in a robust way. We would like to note that the computation of the oscillation of solutions is important for the application to flow in porous media and other transport problems. 
The paper is organized as follows. In the next section we present some basic facts that are used later in the analysis. Section 3 is devoted to the numerical homogenization procedure, its analysis, and the nonlinear multiscale finite element method. The following section is devoted to the two-scale corrector result and its mathematical analysis. In the conclusion we describe how the method can be extended to treat more general elliptic equations.

2. Preliminaries. Let $(\Omega, \Sigma, \mu)$ be a probability space and $L^{p}(\Omega)$ the space of all $p$ integrable functions. Consider a $d$ dimensional dynamical system on $\Omega, T(x)$ : $\Omega \rightarrow \Omega, x \in R^{d}$, that satisfies the following conditions: (1) $T(0)=I$, and $T(x+y)=$ $T(x) T(y)$; (2) $T(x): \Omega \rightarrow \Omega$ preserves the measure $\mu$ on $\Omega$; (3) for any measurable function $f(\omega)$ on $\Omega$, the function $f(T(x) \omega)$ defined on $R^{d} \times \Omega$ is also measurable (e.g., see $[11,17])$.

$U(x) f(\omega)=f(T(x) \omega)$ defines a $d$-parameter group of isometries in the space $L^{p}(\Omega)$, and $U(x)$ is strongly continuous (see [11, p. 223]). Further, we assume that the dynamical system $T$ is ergodic; i.e., any measurable $T$-invariant function on $\Omega$ is constant. Denote by $\langle\cdot\rangle$ the mean value over $\Omega$,

$$
\langle f\rangle=\int_{\Omega} f(\omega) d \mu(\omega)=E(f) .
$$

Denote by $D_{\omega}^{i}$ the generator of $U(x)$ along the $i$ th coordinate direction, i.e.,

$$
D_{\omega}^{i}=\lim _{\delta \rightarrow 0} \frac{f\left(T_{x} e_{i} \omega\right)-f(\omega)}{\delta} .
$$

The domains $\partial_{i}$ of $D_{\omega}^{i}$ are dense in $L^{2}(\Omega)$, and the intersection of all $D_{\omega}^{i}$ is also dense.

Next, following [16, p. 137] (cf. [11]) we define potential and solenoidal fields. A vector field $f \in L^{p}(\Omega)$ is said to be potential (or solenoidal, respectively) if its generic realization $f\left(T_{x} \omega\right)$ is a potential (or solenoidal, respectively) vector field in $R^{d}$. Denote by $L_{\text {pot }}^{p}(\Omega)$ (respectively, $L_{\text {sol }}^{p}(\Omega)$ ) the subspace of $L^{p}(\Omega)$ that consists of all potential (respectively, solenoidal) vector fields. Introduce the following notations:

$$
V_{\text {pot }}^{p}=\left\{f \in L_{\text {pot }}^{p}(\Omega),\langle f\rangle=0\right\}, \quad V_{\text {sol }}^{p}=\left\{f \in L_{\text {sol }}^{p}(\Omega),\langle f\rangle=0\right\} .
$$

The following properties are well known (see [16, p. 138]):

$$
L_{\text {pot }}^{p}(\Omega)=V_{\text {pot }}^{p} \oplus R^{d}, \quad L_{\text {sol }}^{p}(\Omega)=V_{\text {sol }}^{p} \oplus R^{d}, \quad L_{\text {sol }}^{q}(\Omega)=\left(V_{\text {pot }}^{p}\right)^{\perp}, \quad L_{\text {pot }}^{q}(\Omega)=\left(V_{\text {sol }}^{p}\right)^{\perp} .
$$

Consider the equation

$$
\begin{array}{r}
-\operatorname{div}\left(\mathbf{a}\left(T_{x / \epsilon} \omega, D u_{\epsilon}\right)\right)=f \text { in } Q, \\
u_{\epsilon}=0 \text { on } \partial Q,
\end{array}
$$

where $f$ is a deterministic function that does not depend on $\epsilon$ and is sufficiently smooth, and $Q \subset R^{d}$ is a bounded domain with Lipschitz boundaries.

Assume for all $\omega \in \Omega$

$$
\left|\mathbf{a}\left(\omega, \xi_{1}\right)-\mathbf{a}\left(\omega, \xi_{2}\right)\right| \leq C\left(1+\left|\xi_{1}\right|+\left|\xi_{2}\right|\right)^{p-1-\alpha}\left|\xi_{1}-\xi_{2}\right|^{\alpha}
$$

and

$$
\left(\mathbf{a}\left(\omega, \xi_{1}\right)-\mathbf{a}\left(\omega, \xi_{2}\right), \xi_{1}-\xi_{2}\right) \geq C\left(1+\left|\xi_{1}\right|+\left|\xi_{2}\right|\right)^{p-\beta}\left|\xi_{1}-\xi_{2}\right|^{\beta}
$$


For simplicity of analysis we assume $\beta=p, p \geq 2$, and $\mathbf{a}(\omega, 0)=0$. The analysis can be easily generalized.

It is known $[15,16]$ that as $\epsilon \rightarrow 0 D u_{\epsilon}$, converges to $D u$ weakly in $L^{p}(Q)$ for a.e. $\omega$, and $D u$ is the solution of

$$
-\operatorname{div}\left(\mathbf{a}^{*}(D u)\right)=f, \quad u \in W_{0}^{1, p}(Q) .
$$

$\mathbf{a}^{*}$ can be constructed using the solution of the following auxiliary problem. Given $\xi \in R^{d}$ define $w_{\xi} \in V_{\text {pot }}^{p}$ such that

$$
\mathbf{a}\left(\omega, \xi+w_{\xi}(\omega)\right) \in L_{\text {sol }}^{q}(\Omega) .
$$

Then $\mathbf{a}^{*}(\xi)$ is defined as

$$
\mathbf{a}^{*}(\xi)=\left\langle\mathbf{a}\left(\omega, \xi+w_{\xi}(\omega)\right)\right\rangle .
$$

Moreover, $\mathbf{a}^{*}(\xi)$ satisfies the estimates (5) and (6) with new $\alpha$ (see [16, p. 154]). In the first part of the paper we will use general $G$-convergence theory for the analysis. We refer the reader to previous papers $[3,18,16]$ where $G$-convergence for monotone operators is described.

Throughout the paper $C$ denotes a generic constant, $q$ is defined by $1 / p+1 / q=1$, and $\|\cdot\|_{p}$ refers to the $L^{p}(Q)$ norm.

3. Numerical homogenization. In this section we present a numerical procedure for the calculation of the homogenized solution. The analysis of this procedure will be carried out using $G$-convergence theory; i.e., we do not impose any restrictions on the nature of heterogeneities. The proof of the homogeneous random case is simpler and will be addressed. Consider

$$
-\operatorname{div}\left(\mathbf{a}_{\epsilon}\left(x, D u_{\epsilon}\right)\right)=f, \quad u_{\epsilon} \in W_{0}^{1, p}(Q),
$$

where $\epsilon$ is a small scale. The elliptic flux $\mathbf{a}_{\epsilon}(x, \xi)$ satisfies the same assumptions, (5) and (6), imposed in the previous section. We denote the differential operator corresponding to (8) by $A_{\epsilon}$. It is known that (e.g., [16, p. 90]) $u_{\epsilon} \rightarrow u$ and $\mathbf{a}_{\epsilon}\left(x, D u_{\epsilon}\right) \rightarrow \mathbf{a}^{*}(x, D u)$ (up to a subsequence) weakly in $W_{0}^{1, p}(Q)$ and $L^{q}(Q)^{n}$, respectively, and

$$
-\operatorname{div}\left(\mathbf{a}^{*}(x, D u)\right)=f, \quad u \in W_{0}^{1, p}(Q) .
$$

Our numerical homogenization procedure is based on finite element computations on a grid $h$ such that $1 \gg h \gg \epsilon$. To solve a homogenized equation (9) within the framework finite element we seek the solution of (9) in a finite dimensional space over the standard triangulation $K$ of $Q=\bigcup K$,

$S^{h}=\left\{v_{h} \in C^{0}(\bar{Q})\right.$ : the restriction $v_{h}$ is linear for each triangle $K$, and $v_{h}=0$ on $\left.\partial Q\right\}$, such that

$$
\left(A^{*} u_{h}, v_{h}\right)=\int_{Q} f v_{h} d x \forall v \in S^{h},
$$

where

$$
\left(A^{*} u_{h}, v_{h}\right)=\sum_{K} \int_{K}\left(\mathbf{a}^{*}\left(x, D u_{h}\right), D v_{h}\right) d x
$$


The actual computation of $u_{h}$ is carried out by introducing the natural bases in $S^{h}=\operatorname{span}\left(\phi_{i}(x)\right), u_{h}=\sum_{i=1}^{N} \theta_{i} \phi_{i}(x)$. The numerical homogenization procedure approximates $\int_{K} \mathbf{a}^{*}(x, \xi) d x\left(\xi=D u_{h}\right)$ using the solutions of the local problems. In particular, we seek $u_{h, \epsilon}\left(=u_{h}\right) \in S^{h}$ such that

$$
\left(A^{h, \epsilon} u_{h, \epsilon}, v_{h}\right)=\int_{Q} f v_{h} d x \forall v_{h} \in S^{h},
$$

where

$$
\left(A^{h, \epsilon} u_{h, \epsilon}, v_{h}\right)=\sum_{K} \int_{K}\left(\mathbf{a}_{\epsilon}\left(x, D v_{\epsilon}\right), D v_{h}\right) d x
$$

Here $v_{\epsilon}$ is the solution of

$$
\begin{array}{r}
-\operatorname{div}\left(\mathbf{a}_{\epsilon}\left(x, D v_{\epsilon}\right)\right)=0 \text { in } K, \\
v_{\epsilon}=\xi \cdot \mathbf{x} \text { on } \partial K,
\end{array}
$$

where $\xi=$ const $\in R^{d}$ in each $K$ defined by $\xi=D u_{h, \epsilon}$. Here and later we will denote by $\xi=$ const $\in R^{d}$ (const in each $K$ ) the gradient of an element $u_{h} \in S^{h}$ in each $K, \xi=D u_{h}$. Equation (11) is our numerical homogenization procedure whose convergence will be studied next. We would like to note that as a boundary condition in (13) one can also take $v_{\epsilon}=u_{h, \epsilon}(=\xi \cdot x+c)$ (a linear function) on $\partial K$. The discrete formulation of the numerical homogenization procedure is as follows. We seek the solution $u_{h}=\sum_{i=1}^{N} \theta_{i} \phi_{i}(x)$ such that

$$
\mathbf{F}(\theta)=\mathbf{b},
$$

where $\theta=\left(\theta_{1}, \ldots, \theta_{N}\right), b_{i}=\int_{Q} f \phi_{i}(x) d x, F_{i}(\theta)=\sum_{K} \int_{K}\left(\mathbf{a}_{\epsilon}\left(x, D v_{\epsilon}\right), D \phi_{i}(x)\right) d x$, $i=1, \ldots, N$, and $v_{\epsilon}$ satisfies (13) with boundary conditions $v_{\epsilon}=\sum_{i} \theta_{i} \phi_{i}(x)$ on $\partial K$ (or $v_{\epsilon}=\xi \cdot x$, where $\xi$ is a constant which is equal to $D \sum_{i} \theta_{i} \phi_{i}(x)$ in $K$ ). The implementation of the numerical homogenization procedure will also be discussed.

TheOREM 3.1. $u_{h, \epsilon} \rightarrow u$ in $W_{0}^{1, p}(Q)$ (up to a subsequence of $\epsilon$ ) as $\lim _{h \rightarrow 0} \lim _{\epsilon \rightarrow 0}$, under some additional smoothness assumptions on $\mathbf{a}^{*}(x, \xi)$ discussed below. Here $u_{h, \epsilon}$ is the solution of (11) and $u$ is the solution of (9).

Remark. We note that within the theory of $G$-convergence of monotone operators the limit operator (9) may be nonunique. Thus, in Theorem 3.1 we have a convergence up to a subsequence to the solution of a homogenized equation. In the case of the random homogeneous elliptic flux the limiting homogenization equation is unique and, thus, the whole sequence converges.

To prove the theorem we will first investigate the limit of (11) as $\epsilon \rightarrow 0$ and then as $h \rightarrow 0$. For the proof we need the trace-free analogue of $v_{\epsilon}$, the solution of (13), $v_{\epsilon}^{b} \in W_{0}^{1, p}(K)$, that satisfies

$$
\operatorname{div}\left(\mathbf{a}_{\epsilon}\left(x, \xi+D v_{\epsilon}^{b}\right)\right)=0
$$

where $\xi=D u_{h}$ (cf. (13)) and $u_{h}$ is an element of $S^{h}$.

Lemma 3.2. $A^{h, \epsilon}$ is strictly monotone for any $h>0$.

Proof. For any $u_{h}^{1} \in S^{h}$ and $u_{h}^{2} \in S^{h}$ we have 


$$
\begin{array}{r}
\left(A^{h, \epsilon} u_{h}^{1}-A^{h, \epsilon} u_{h}^{2}, u_{h}^{1}-u_{h}^{2}\right)=\sum_{K} \int_{K}\left(\mathbf{a}_{\epsilon}\left(x, D v_{\epsilon}^{1}\right)-\mathbf{a}_{\epsilon}\left(x, D v_{\epsilon}^{2}\right), D u_{h}^{1}-D u_{h}^{2}\right) d x \\
=\sum_{K} \int_{K}\left(\mathbf{a}_{\epsilon}\left(x, \xi_{1}+D v_{\epsilon}^{1, b}\right)-\mathbf{a}_{\epsilon}\left(x, \xi_{2}+D v_{\epsilon}^{2, b}\right), \xi_{1}-\xi_{2}\right) d x \\
=\sum_{K} \int_{K}\left(\mathbf{a}_{\epsilon}\left(x, \xi_{1}+D v_{\epsilon}^{1, b}\right)-\mathbf{a}_{\epsilon}\left(x, \xi_{2}+D v_{\epsilon}^{2, b}\right), D v_{\epsilon}^{1, b}+\xi_{1}-\left(D v_{\epsilon}^{2, b}+\xi_{2}\right)\right) d x \\
\geq C \sum_{K} \int_{K}\left|\xi_{1}+D v_{\epsilon}^{1, b}-\left(\xi_{2}+D v_{\epsilon}^{2, b}\right)\right|^{p} d x=C \sum_{K} \int_{K}\left|D v_{\epsilon}^{1}-D v_{\epsilon}^{2}\right|^{p} d x
\end{array}
$$

Here $v_{\epsilon}^{1}$ and $v_{\epsilon}^{2}$ satisfy (13) with $\xi_{1}=D u_{h}^{1}$ and $\xi_{2}=D u_{h}^{2}$, and $v_{\epsilon}^{1, b}=v_{\epsilon}^{1}-u_{h}^{1}$ and $v_{\epsilon}^{2, b}=v_{\epsilon}^{2}-u_{h}^{2}$. Taking into account that $D v_{\epsilon}^{1}=D v_{\epsilon}^{2}$ if and only if $\xi_{1}=\xi_{2}$ we obtain strict monotonicity.

Lemma 3.3. $A^{h, \epsilon}$ is coercive for any $h>0$.

Proof. Indeed, for any $u_{h} \in S^{h}$

$$
\begin{array}{r}
\left(A^{h, \epsilon} u_{h}, u_{h}\right)=\sum_{K} \int_{K}\left(\mathbf{a}_{\epsilon}\left(x, D v_{\epsilon}\right), \xi\right) d x=\sum_{K} \int_{K}\left(\mathbf{a}_{\epsilon}\left(x, \xi+D u_{\epsilon}\right), \xi\right) d x \\
\geq C \sum_{K} \int_{K}\left|\xi+D u_{\epsilon}\right|^{p} d x=C \sum_{K} \int_{K}\left|D v_{\epsilon}\right|^{p} d x .
\end{array}
$$

If $\mathbf{a}_{\epsilon}(x, \xi)$ is an homogeneous function with respect to $\xi$, i.e., $\mathbf{a}_{\epsilon}(x, \lambda \xi)=\lambda^{p-1} \mathbf{a}_{\epsilon}(x, \xi)$, then it can be easily shown using a rescaling argument that $\left\|D v_{\epsilon}\right\|_{p}=C\|\xi\|^{p}$. In general we use the trace inequality (see $[14,12])\|u\|_{p, \partial K} \leq C\|D u\|_{p, K}$ to obtain lower bound for (17). Denote $K_{1}$ to be rescaled $K$ such that $\operatorname{diam}\left(K_{1}\right)=O(1), y=x / h$, $v_{\epsilon}^{1}=v_{\epsilon}(y h)$. Then

$$
\begin{array}{r}
\int_{K}\left|D v_{\epsilon}\right|^{p} d x=C \frac{h^{d}}{h^{p}} \int_{K_{1}}\left|D_{y} v_{\epsilon}^{1}\right|^{p} d y \geq C \frac{h^{d}}{h^{p}} \int_{\partial K_{1}}\left|v_{\epsilon}^{1}\right|^{p} d S_{y}=C h^{d} \int_{\partial K_{1}}|\xi \cdot x|^{p} d S_{y} \\
\geq C|\xi|^{p} h^{d}=C \int_{K}|\xi|^{p} d x
\end{array}
$$

Here we have used the fact that the norms in finite dimensional spaces are equivalent, and $\int_{\partial K_{1}}|\xi \cdot x| d S_{y}$ generates a norm in the corresponding finite dimensional space of $\xi$ 's. Note that since we use the equivalence of norms in a finite dimensional space the constant $C$ in (18) depends on $h$ in general. Thus,

$$
\left(A^{h, \epsilon} u_{h}, u_{h}\right) \geq C\left\|D u_{h}\right\|_{L^{p}(Q)}^{p} .
$$

Strict monotonicity and coercivity guarantee the existence and uniqueness of the solution $u_{h, \epsilon}$ of (11) for any $h>0$.

LEMma 3.4. For any $u_{h} \in S^{h}$ we have

$$
\lim _{\epsilon \rightarrow 0} A^{h, \epsilon} u_{h}=A^{h} u_{h},
$$

where $A^{h}$ is defined on $S^{h}$ and given by

$$
\left(A^{h} u_{h}, v_{h}\right)=\sum_{K} \int_{K}\left(\mathbf{a}^{*}\left(x, D v_{0}\right), D v_{h}\right) d x .
$$


Here $v_{0}$ is the solution of

$$
-\operatorname{div}\left(\mathbf{a}^{*}\left(x, D v_{0}\right)\right)=0 \text { in } K,
$$

$v_{0}=\xi \cdot x$ on $\partial K, \xi=D u_{h}$.

Proof. The proof follows from the convergence of arbitrary solutions of the $G$ convergent sequence of operators. Assume $\mathbf{a}^{*}(x, \xi)$ is a flux for a $G$-limit of $A_{\epsilon}$ (up to a subsequence). We call this subsequence the original subsequence in further analysis. Then from (13) and the local nature of the $G$-converging sequence (e.g., [16, p. 96],) it follows that $v_{\epsilon} \rightarrow v_{0}$ (up to a subsequence) weakly in $W^{1, p}(K)$ as $\epsilon \rightarrow 0$, where $v_{0}$ is the solution of (20). Moreover,

$$
\mathbf{a}_{\epsilon}\left(x, D v_{\epsilon}\right) \rightarrow \mathbf{a}^{*}\left(x, D v_{0}\right)
$$

weakly in $L^{q}(K)^{n}$ as $\epsilon \rightarrow 0$. Since the solution $v_{0}$ is unique the whole sequence (of the original subsequence) converges. Using the latter and taking into account the form of $A^{h, \epsilon}$ we obtain the desired result by taking the limit as $\epsilon \rightarrow 0$ of (12).

Next, we show that the above convergence in Lemma 3.4 is uniform in a compact set. For this it is sufficient to show that $A^{h, \epsilon}$ is equicontinuous since the problem is finite dimensional.

LEMMA 3.5.

$$
\left\|A^{h, \epsilon} u_{h}^{1}-A^{h, \epsilon} u_{h}^{2}\right\| \leq C\left\|u_{h}^{1}-u_{h}^{2}\right\|^{\gamma},
$$

with $\gamma>0$, for any $u_{h}^{1}$ and $u_{h}^{2}$ in a compact set. The constant $C$ is independent of $\epsilon$.

Remark. Both norms are considered in finite dimensional space; thus we do not specify them.

Proof. Clearly, for any $u_{h}^{1} \in S^{h}$ and $u_{h}^{2} \in S^{h}$ we have

$$
\begin{aligned}
& \left\|A^{h, \epsilon} u_{h}^{1}-A^{h, \epsilon} u_{h}^{2}\right\| \\
& \leq\left|\sum_{K} \int_{K}\left(\mathbf{a}_{\epsilon}\left(x, \xi_{1}+D v_{\epsilon}^{1, b}\right)-\mathbf{a}_{\epsilon}\left(x, \xi_{2}+D v_{\epsilon}^{2, b}\right)\right) d x\right| \\
& \leq C \sum_{K} \int_{K}\left(1+\left|D v_{\epsilon}^{1}\right|^{p-1-s}+\left|D v_{\epsilon}^{2}\right|^{p-1-s}\right)|| \xi_{1}+D v_{\epsilon}^{1, b}-\left.\left(\xi_{2}+D v_{\epsilon}^{2, b}\right)\right|^{s} d x \\
& \leq C\left(\sum_{K} \int_{K}\left|\xi_{1}+D v_{\epsilon}^{1, b}-\left(\xi_{2}+D v_{\epsilon}^{2, b}\right)\right|^{p} d x\right)^{1 / p} \\
& \leq C\left(\sum_{K} \int_{K}\left(\mathbf{a}\left(x, \frac{x}{\epsilon}, \xi_{1}+D v_{\epsilon}^{1, b}\right)-\mathbf{a}\left(x, \frac{x}{\epsilon}, \xi_{2}+D v_{\epsilon}^{2, b}\right), \xi_{1}+D v_{\epsilon}^{1, b}-\xi_{2}-D v_{\epsilon}^{2, b}\right) d x \mid\right)^{1 / p} \\
& =C\left(\sum_{K} \int_{K}\left(\mathbf{a}\left(x, \frac{x}{\epsilon}, \xi_{1}+D v_{\epsilon}^{1, b}\right)-\mathbf{a}\left(x, \frac{x}{\epsilon}, \xi_{2}+D v_{\epsilon}^{2, b}\right), \xi_{1}-\xi_{2}\right) d x \mid\right)^{1 / p} \\
& \leq C\left(\sum_{K} \int_{K}\left|\xi_{1}-\xi_{2}\right|^{p} d x\right)^{1 / p^{2}} \leq C\left(\int_{Q}\left|D u_{h}^{1}-D u_{h}^{2}\right|^{p} d x\right)^{1 / p^{2}},
\end{aligned}
$$

where $v_{\epsilon}^{i}=v_{\epsilon}^{i, b}+u_{h}^{i}(i=1,2)$ are local solutions (see (13) and (15)) and $\xi_{i}=D u_{h}^{i}$ $(i=1,2)$. Here we have used the fact that $D v_{\epsilon}^{1}$ and $D v_{\epsilon}^{2}$ are bounded in $L^{p}$. Indeed, 
it can be easily derived that $\left\|D v_{\epsilon}^{i}\right\|_{L^{p}(Q)} \leq C\left\|D u_{h}^{i}\right\|_{L^{p}(Q)}(i=1,2)$, and since $u_{h}^{i}$ is assumed to be in a compact set, thus $\left\|D v_{\epsilon}^{i}\right\|_{L^{p}(Q)} \leq C(i=1,2)$.

From the coercivity of $A^{h, \epsilon}$ we obtain that $u_{h, \epsilon} \rightarrow u_{h}$ (finite dimensional) as $\epsilon \rightarrow 0$ (up to a subsequence) for any $h>0$. Taking the limit as $\epsilon \rightarrow 0$ of (11) and using Lemmas 3.4 and 3.5 we get

$$
\left(A^{h} u_{h}, v_{h}\right)=\int_{Q} f v_{h} d x \forall v_{h} \in S^{h}
$$

where $A^{h}$ is defined by (19). We can easily show that (see below) the solution of this discrete equation exists and is unique; thus the whole sequence converges. Note that this whole sequence is a subsequence of the $G$-convergent sequence discussed in Lemma 3.4.

We note that if the homogenized operator does not depend on the spatial variables, then the proof of the Theorem 3.1 is complete. Indeed, in this case $v_{0}=u_{h}$, and thus $A^{h}=A^{*}$, where $A^{*}$ is defined by

$$
\left(A^{*} u_{h}, v_{h}\right)=\sum_{K} \int_{K}\left(\mathbf{a}^{*}\left(x, D u_{h}\right), D v_{h}\right) d x
$$

Thus the discrete equation $\left(A^{h} u_{h}, v_{h}\right)=\left(A^{*} u_{h}, v_{h}\right)=\int_{Q} f v_{h}$ is a standard Galerkin discretization of a homogenized equation, and, consequently, $u_{h}$ converges to $u$ in $W_{0}^{1, p}(Q)$ as $h \rightarrow 0$. If $\mathbf{a}^{*}(x, \xi)$ has spatial dependence, then $A^{h}$ differs from $A^{*}$. Our further goal is to study the limit as $h \rightarrow 0$. For this reason we need to assume some smoothness for $\mathbf{a}^{*}(x, \xi)$ with respect to spatial variables. Introduce $\overline{\mathbf{a}}^{*}(\xi)=$ $\frac{1}{|K|} \int_{K} \mathbf{a}^{*}(x, \xi) d x$. Clearly $\operatorname{div}\left(\overline{\mathbf{a}}\left(D u_{h}\right)\right)=0$. Assume that $\left|\overline{\mathbf{a}}^{*}(\xi)-\mathbf{a}^{*}(x, \xi)\right| \leq C \alpha_{h}(1+$ $|\xi|^{p-1}$ ), where $\alpha_{h}$ is a generic sequence (independent of $x$ ), such that $\alpha_{h} \rightarrow 0$ as $h \rightarrow 0$. The latter is true, for example, if $\mathbf{a}^{*}(x, \xi)$ is Hölder continuous with respect to spatial variables. We do not know how to show it (Hölder continuity of $\mathbf{a}^{*}(x, \xi)$ ), in general, based on the smoothness properties of $\mathbf{a}_{\epsilon}(x, \xi)$, and this is currently under investigation.

For the following analysis we note that $\mathbf{a}^{*}$ satisfies assumptions such as (5) and (6) with possibly different constant in (5) (e.g., see [16, p. 86]):

$$
\left|\mathbf{a}^{*}\left(x, \xi_{1}\right)-\mathbf{a}^{*}\left(x, \xi_{2}\right)\right| \leq C\left(1+\left|\xi_{1}\right|+\left|\xi_{2}\right|\right)^{p-1-\bar{\alpha}}\left|\xi_{1}-\xi_{2}\right|^{\bar{\alpha}} .
$$

LEMma 3.6. $A^{h}$ (defined by (19)) is coercive for sufficiently small $h$.

Proof. For any $u_{h} \in S^{h}$ we have

$$
\begin{aligned}
\left(A^{h} u_{h}, u_{h}\right) & =\sum_{K} \int_{K}\left(\mathbf{a}^{*}\left(x, D v_{0}\right), D u_{h}\right) d x \\
& =\sum_{K} \int_{K}\left(\mathbf{a}^{*}\left(x, D v_{0}\right), D u_{h}+D v_{0}^{b}\right) d x \\
& \geq C\left\|D v_{0}\right\|_{L^{p}(Q)}^{p},
\end{aligned}
$$


where $v_{0}$ satisfies (20). Next, we estimate $D v_{0}$.

$$
\begin{aligned}
\left\|D v_{0}-D u_{h}\right\|_{L^{p}(Q)}^{p} \\
\leq C \sum_{K} \int_{K}\left(\mathbf{a}^{*}\left(x, D v_{0}\right)-\mathbf{a}^{*}\left(x, D u_{h}\right), D v_{0}-D u_{h}\right) d x \\
=C \sum_{K} \int_{K}\left(\mathbf{a}^{*}\left(x, D v_{0}\right)-\overline{\mathbf{a}}^{*}\left(D u_{h}\right), D v_{0}-D u_{h}\right) d x \\
\quad+C \sum_{K} \int_{K}\left(\overline{\mathbf{a}}^{*}\left(D u_{h}\right)-\mathbf{a}^{*}\left(x, D u_{h}\right), D v_{0}-D u_{h}\right) d x \\
=C \sum_{K} \int_{K}\left(\overline{\mathbf{a}}^{*}\left(D u_{h}\right)-\mathbf{a}^{*}\left(x, D u_{h}\right), D v_{0}-D u_{h}\right) d x \\
\leq C_{\delta} \alpha_{h}\left(1+\left\|D u_{h}\right\|_{L^{p}(Q)}^{p}\right)+C \delta\left\|D v_{0}-D u_{h}\right\|_{L^{p}(Q)}^{p} .
\end{aligned}
$$

With an appropriate choice of $\delta$ and for sufficiently small $h$ we obtain

$$
\left\|D v_{0}\right\|_{L^{p}(Q)}^{p} \geq C\left\|D u_{h}\right\|_{L^{p}(Q)}^{p}-C \alpha_{h} .
$$

It can be easily shown that (as in Lemma 3.2 ) $A^{h}$ is strongly monotone, which implies that the solution $u_{h}$ of (23) is unique. Note that from the coercivity of $A^{h}$ we obtain that $\left\|D u_{h}\right\|_{p, Q} \leq C$, where $C$ is independent of $h$. Next, we study the approximation properties of $A^{h}$.

Lemma 3.7. $\left(A^{h} u_{h}-A^{*} u_{h}, w_{h}\right) \rightarrow 0$ as $h \rightarrow 0$ for any families of $u_{h} \in S^{h}$ and $w_{h} \in S^{h}$ uniformly bounded in $W_{0}^{1, p}(Q)$, where $A^{h}$ and $A^{*}$ are defined by (19) and (24), respectively.

Proof.

$$
\begin{array}{r}
\left(A^{h} u_{h}-A^{*} u_{h}, D w_{h}\right)=\sum_{K} \int_{K}\left(\mathbf{a}^{*}\left(x, D v_{0}\right)-\mathbf{a}^{*}\left(x, D u_{h}\right), D w_{h}\right) d x \\
\leq C\left(1+\left\|D v_{0}\right\|_{p, Q}^{(p-q \bar{\alpha}) / q}+\left\|D u_{h}\right\|_{p, Q}^{(p-q \bar{\alpha} / q}\right)\left\|D v_{0}-D u_{h}\right\|_{p, Q}^{\bar{\alpha}}\left\|D w_{h}\right\|_{p, Q} \\
\leq C \alpha_{h}\left(\int_{Q}\left(1+\left|D u_{h}\right|^{p}\right) d x\right)^{\bar{\alpha} / p}\left(\int_{Q}\left(\left|D w_{h}\right|^{p}\right) d x\right)^{1 / p} .
\end{array}
$$

In the last step we have used (27). Clearly, the right-hand side (r.h.s.) converges to zero as $h \rightarrow 0$ for any uniformly bounded family of $u_{h}$ and $w_{h}$ in $W_{0}^{1, p}(Q)$.

To finish the proof of the Theorem 3.1 we introduce $P_{h} u$, the orthogonal projection of the solution of (9) onto $S^{h}$. Clearly, $P_{h} u \rightarrow u$ in $W_{0}^{1, p}(Q)$ as $h \rightarrow 0$. Then

$$
\begin{array}{r}
\left\|D u_{h}-D P_{h} u\right\|_{L^{p}(Q)}^{p} \leq C\left(A^{*} u_{h}-A^{*} P_{h} u, u_{h}-P_{h} u\right)=C\left(A^{*} u_{h}-A^{h} u_{h}, u_{h}-P_{h} u\right) \\
+C\left(A^{h} u_{h}-A^{*} P_{h} u, u_{h}-P_{h} u\right) \leq \alpha_{h}+C\left(f-A^{*} P_{h} u, u_{h}-P_{h} u\right) \\
=\alpha_{h}+C\left(A^{*} u-A^{*} P_{h} u, u_{h}-P_{h} u\right) .
\end{array}
$$

Here we have used Lemma 3.7, where $\alpha_{h}$ is a generic sequence, $\alpha_{h} \rightarrow 0$, as $h \rightarrow 0$, and the facts that $\left(A^{h} u_{h}, v_{h}\right)=\left(f, v_{h}\right)=\left(A^{*} u, v_{h}\right)$, for all $v_{h} \in S^{h}$, and both $D P_{h} u$ and $D u_{h}$ are uniformly bounded in $L^{p}(Q)$. The second term converges to zero because $A^{*}$ is continuous. Thus, $\left\|D u_{h}-D P_{h} u\right\|_{L^{p}(Q)} \rightarrow 0$ as $h \rightarrow 0$. This completes the proof of Theorem 3.1. 
Next we make some comments on the use of Newton's methods (e.g., see [20]) for solving the nonlinear equation (11). The discrete formulation of this equation is $\mathbf{F}(\theta)=\mathbf{b}$ (see (14)). To solve this system using Newton's method we do $\theta^{n+1}=$ $\theta^{n}-\left(\mathbf{F}^{\prime}\left(\theta^{n}\right)\right)^{-1} \mathbf{F}\left(\theta^{n}\right)$. The corresponding linearized system $B_{i j}$,

$$
B_{i j}=\frac{\partial}{\partial \theta_{j}} F_{i}(\theta)
$$

is sparse. Note that the convergence of Newton's method requires additional assumptions on $\mathbf{a}^{*}(x, \xi)$, such as smoothness with respect to $\xi$. We do not know how to show it based on the smoothness of $\mathbf{a}_{\epsilon}(x, \xi)$. Thus, in applying Newton's method we need to assume that $\mathbf{a}^{*}(x, \xi)$ is continuously differentiable with respect to $\xi$.

In order to carry out an iteration of Newton's method for a given $\theta_{i}$ we need to compute $\mathbf{F}(\theta)$ and $B_{i j}$. The latter requires the computation of $\int_{K} \mathbf{a}^{*}(x, \xi) d x$ for a given constant $\xi \in R^{d}$ and its derivatives with respect to $\theta$. The approximation of $\int_{K} \mathbf{a}^{*}(x, \xi) d x$ is computed as shown above using the local solution of (13). We would like to note that because of strict monotonicity of $\mathbf{F}(\theta) B_{i j}$ is nonsingular. Consequently, Newton's method is applicable, provided $\mathbf{a}^{*}(x, \xi)$ is continuously differentiable with respect to $\xi$.

Note that in the periodic case one can solve a cell problem for each $\xi$ and $x$ and compute $\mathbf{a}^{*}$. As can be seen, our numerical procedure has some advantage even in the periodic case because we compute only $\mathbf{a}^{*}(x, \xi)$ for some values of $\xi$ that are needed in the iterative procedure. Furthermore, the matrix $B_{i j}$ is defined on the coarse grid; thus its inversion can be calculated quickly. The latter is similar to the numerical homogenization of linear equations where the resulting stiffness matrix is defined on the coarse grid.

It is often advantageous to solve the local problem in a larger domain (larger than $K)$ in order to reduce the boundary effects. In this case to show the convergence of the numerical procedure the following theorem on the convergence of arbitrary solutions (e.g., see [16]) can be used.

Theorem 3.8. Assume $A_{\epsilon} \rightarrow^{G} A^{*}$ in $Q, Q_{1} \subset Q, u_{\epsilon} \rightarrow u_{0}$ weakly in $W^{1, p}\left(Q_{1}\right)$, $-\operatorname{div}\left(\left(\mathbf{a}_{\epsilon}\left(x, \xi+D u_{\epsilon}\right)\right)=0\right.$. Then $\mathbf{a}_{\epsilon}\left(x, \xi+D u_{\epsilon}\right) \rightarrow \mathbf{a}^{*}\left(x, \xi+D u_{0}\right)$ in $L^{q}\left(Q_{1}\right)$ and $-\operatorname{div}\left(\mathbf{a}^{*}\left(x, \xi+D u_{0}\right)\right)=0$.

In the periodic case one can use instead of the elements $K$ a period of size $\epsilon$ as it is done in HMM [6].

3.1. Nonlinear multiscale finite element methods (NMsFEM). The numerical homogenization procedure presented above can be put in a framework of finite elements as it is done in the case of multiscale finite element methods [9]. For multiscale finite element methods the space that contains the small-scale information of the solution is a linear space. The latter is no longer the case for the nonlinear equations. Consider a standard finite dimensional space $S^{h}$ over the coarse triangulation of $Q$, where the base functions $\phi_{0}^{i}$ are linear functions on each triangle. Define a nonlinear space, $V_{\epsilon}^{h}$, of functions over the coarse triangulation whose elements are the solutions of

$$
\operatorname{div}\left(\mathbf{a}_{\epsilon}\left(x, D \phi_{\epsilon}^{i}\right)\right)=0 \text { in } K,
$$

with boundary conditions $\phi_{\epsilon}^{i}=\phi_{0}^{i}$ on $\partial K$. Clearly, the sum of two elements of $V_{\epsilon}^{h}$ does not belong to $V_{\epsilon}^{h}$. For each two elements $\phi_{\epsilon}^{i}$ and $\phi_{\epsilon}^{j}$, we define their sum $\phi_{\epsilon}^{i, j}=\phi_{\epsilon}^{i}+\phi_{\epsilon}^{j}$ as the solution of

$$
\operatorname{div}\left(\mathbf{a}_{\epsilon}\left(x, D \phi_{\epsilon}^{i, j}\right)\right)=0 \text { in } K
$$


with boundary conditions $\phi_{\epsilon}^{i, j}=\phi_{0}^{i}+\phi_{0}^{j}$ on $\partial K$. Clearly, with such summation rule each element of $v_{h, \epsilon} \in V_{\epsilon}^{h}$ can be represented as $v_{h, \epsilon}=\sum_{i} \theta_{i} \phi_{\epsilon}^{i}$, and there is one-toone nonlinear operator $E$ such that $E: S^{h} \rightarrow V_{\epsilon}^{h}$. In particular, for each $v_{h} \in S^{h}$ a corresponding element of $v_{h, \epsilon} \in V_{\epsilon}^{h}$ solves $\operatorname{div}\left(\mathbf{a}_{\epsilon}\left(x, D v_{h, \epsilon}\right)\right)=0$ in each $K$ subject to boundary conditions $v_{h, \epsilon}=v_{h}$ on $\partial K$.

Next we propose the Petrov-Galerkin formulation of (8). Find $u_{h} \in V_{\epsilon}^{h}$ such that

$$
A\left(u_{h}, v_{h}\right)=\int_{Q} f v_{h} d x \forall v_{h} \in S^{h},
$$

where

$$
A\left(u_{h}, v_{h}\right)=\int_{Q}\left(\mathbf{a}_{\epsilon}\left(x, D v_{\epsilon}\right), D v_{h}\right) d x
$$

and $v_{\epsilon}$ is the solution of (13). This formulation is equivalent to the numerical homogenization procedure described previously. Consequently, $u_{h}$ converges (up to a subsequence, in general, and the whole sequence in the case of an homogeneous random field) to a homogenized solution of the original equation. Next, we will show that $u_{h}=\sum_{i} \theta_{i} \phi_{\epsilon}^{i}$ approximates the gradient of the solution of original equation under the assumption that the elliptic flux is strictly stationary with respect to spatial variables.

4. Two-scale corrector. An approximation of the solution gradient. In this section we show how the gradient of the solution can be approximated for the case of spatially homogeneous elliptic fluxes, (4). The importance of this approximation is motivated by some applications where details of the fluxes play a key role in a physical phenomenon (e.g., flow in porous media). For the construction we assume that the homogenized solution is computed with a reliable accuracy as in the previous section. Our construction borrows its main idea from [4], which is for periodic operators. The distinction is that we introduce two scales in the construction where one scale represents the numerical scale $h$ and the other the physical scale $\epsilon$.

Define $M_{h} \phi(x)$ in the following way:

$$
M_{h} \phi(x)=\sum_{i} 1_{Q_{i}} \frac{1}{\left|Q_{i}\right|} \int_{Q_{i}} \phi(y) d y,
$$

where $\bigcup Q_{i}=Q$. Here $Q_{i}$ are domains with diameter of order $h$, e.g., finite element triangles or some unions of the triangles. Note that $M_{h} \phi \rightarrow \phi$ in $L^{p}(Q)$ as $h \rightarrow 0$ (also a.e. in $Q$; e.g., see [22]). Further, denote

$$
P\left(T_{y} \omega, \xi\right)=\xi+w_{\xi}\left(T_{y} \omega\right),
$$

where $w_{\xi} \in L_{p o t}^{p}(\Omega)$ is the solution of auxiliary problem $\mathbf{a}\left(\omega, \xi+w_{\xi}(\omega)\right) \in L_{\text {sol }}^{q}(\Omega)$, or

$$
-\operatorname{div}\left(\mathbf{a}\left(T_{y} \omega, \xi+w_{\xi}\left(T_{y} \omega\right)\right)\right)=0
$$

in the sense of distribution.

One of our main results is the following theorem.

THEOREM 4.1.

$$
\lim _{h \rightarrow 0} \lim _{\epsilon \rightarrow 0} \int_{\Omega} \int_{Q}\left|P\left(T_{x / \epsilon} \omega, M_{h} D u\right)-D u_{\epsilon}\right|^{p} d x d \mu(\omega) \rightarrow 0,
$$


where $u_{\epsilon}$ and $u$ are solutions of (4) and (7), respectively.

The result indicates that the gradient of the solution can be approximated by $P\left(T_{x / \epsilon} \omega, M_{h} D u\right)$. This quantity can be computed based on $M_{h} D u$, i.e., the gradient of the coarse solution in each coarse block, as we will show later.

For the proof of the Theorem 4.1 we need the following lemma.

Lemma 4.2. For every $\xi \in R^{d}$

$$
\int_{\Omega}|P(\omega, \xi)|^{p} d \mu(\omega) \leq C(1+|\xi|)^{p} .
$$

Proof. From monotonicity of a we obtain

$$
\begin{array}{r}
\int_{\Omega}|P(\omega, \xi)|^{p} d \mu(\omega) \leq C \int_{\Omega}(\mathbf{a}(\omega, P)-\mathbf{a}(\omega, 0), P) d \mu(\omega) \\
=\int_{\Omega}(\mathbf{a}(\omega, P), P) d \mu(\omega)=\int_{\Omega}(\mathbf{a}(\omega, P), \xi) d \mu(\omega) \leq C \eta\|P\|_{p}^{p}+C \eta^{-1 /(p-1)}\left(1+|\xi|^{p}\right) .
\end{array}
$$

With an appropriate choice of $\eta$ we obtain the desired result.

From here it follows that $P\left(T_{y} \omega, \xi\right) \in L_{l o c}^{p}\left(R^{d}\right)$ for a.e. $\omega$.

THEOREM 4.3 .

$$
\begin{array}{r}
\lim _{\epsilon \rightarrow 0} \int_{\Omega} \int_{Q}\left|P\left(T_{x / \epsilon} \omega, M_{h} D u\right)-D u_{\epsilon}\right|^{p} d x d \mu(\omega) \\
\leq C\left[\int_{Q}\left(\mathbf{a}^{*}\left(M_{h} D u\right), M_{h} D u\right) d x-\int_{Q}\left(\mathbf{a}^{*}\left(M_{h} D u\right), D u\right) d x-\int_{Q}\left(\mathbf{a}^{*}(D u), M_{h} D u\right) d x\right. \\
\left.+\int_{Q}\left(\mathbf{a}^{*}(D u), D u\right) d x\right] .
\end{array}
$$

The proof of the theorem will be based on the following estimate:

$$
\begin{aligned}
& \int_{\Omega} \int_{Q}\left|P\left(T_{x / \epsilon} \omega, M_{h} D u\right)-D u_{\epsilon}\right|^{p} d x d \mu(\omega) \\
& \leq C \int_{\Omega} \int_{Q}\left(\mathbf{a}\left(T_{x / \epsilon} \omega, P\left(T_{x / \epsilon} \omega, M_{h} D u\right)\right)-\mathbf{a}\left(T_{x / \epsilon} \omega, D u_{\epsilon}\right), P\left(T_{x / \epsilon} \omega, M_{h} D u\right)-D u_{\epsilon}\right) d x d \mu(\omega) \\
& =C \int_{\Omega} \int_{Q}\left(\mathbf{a}\left(T_{x / \epsilon} \omega, P\left(T_{x / \epsilon} \omega, M_{h} D u\right)\right), P\left(T_{x / \epsilon} \omega, M_{h} D u\right)\right) d x d \mu(\omega) \\
& -C \int_{\Omega} \int_{Q}\left(\mathbf{a}\left(T_{x / \epsilon} \omega, P\left(T_{x / \epsilon} \omega, M_{h} D u\right)\right), D u_{\epsilon}\right) d x d \mu(\omega) \\
& -C \int_{\Omega} \int_{Q}\left(\mathbf{a}\left(T_{x / \epsilon} \omega, D u_{\epsilon}\right), P\left(T_{x / \epsilon} \omega, M_{h} D u\right)\right) d x d \mu(\omega) \\
& \quad+C \int_{\Omega} \int_{Q}\left(\mathbf{a}\left(T_{x / \epsilon} \omega, D u_{\epsilon}\right), D u_{\epsilon}\right) d x d \mu(\omega) .
\end{aligned}
$$

We will investigate the r.h.s. of (34) in the limit as $\epsilon \rightarrow 0$.

For the first term of the r.h.s. of (34) we have the following. 
LEMMA 4.4.

$\int_{\Omega} \int_{Q}\left(\mathbf{a}\left(T_{x / \epsilon} \omega, P\left(T_{x / \epsilon} \omega, M_{h} D u\right)\right), P\left(T_{x / \epsilon} \omega, M_{h} D u\right)\right) d x d \mu(\omega) \rightarrow \int_{Q}\left(\mathbf{a}^{*}\left(M_{h} D u\right), M_{h} D u\right) d x$ as $\epsilon \rightarrow 0$.

Proof.

$$
\begin{array}{r}
\int_{\Omega} \int_{Q}\left(\mathbf{a}\left(T_{x / \epsilon} \omega, P\left(T_{x / \epsilon} \omega, M_{h} D u\right)\right),\right. \\
\left.=\sum_{i} \int_{\Omega} \int_{Q_{i}}\left(T_{x / \epsilon} \omega, M_{h} D u\right)\right) d x d \mu(\omega) \\
\rightarrow \sum_{i} \int_{Q_{i}} 1_{Q_{i}}\left(\mathbf{a}^{*}\left(\xi_{i}\right), \xi_{i}\right) d x
\end{array}
$$

as $\epsilon \rightarrow 0$. In the last step we have used the Birkhoff ergodic theorem (see [11, p. 225]), and the fact that $\left\langle\left(\mathbf{a}\left(\omega, \xi+w_{\xi}\right), w_{\xi}\right)\right\rangle=0$, because of $w_{\xi} \in V_{\text {pot }}^{p}, \mathbf{a}\left(\omega, \xi+w_{\xi}\right) \in L_{\text {sol }}^{q}(\Omega)$, and $L_{\text {sol }}^{q}(\Omega)=\left(V_{\text {pot }}^{p}\right)^{\perp}$ (see (3)). Next we note that the limit can be written as

$$
\sum_{i} \int_{Q_{i}} 1_{Q_{i}}\left(\mathbf{a}^{*}\left(\xi_{i}\right), \xi_{i}\right) d x=\int_{Q}\left(\mathbf{a}^{*}\left(M_{h} D u\right), M_{h} D u\right) d x .
$$

For the second term of the r.h.s. of (34) we have the following.

LEMMA 4.5.

$$
\int_{\Omega} \int_{Q}\left(\mathbf{a}\left(T_{x / \epsilon} \omega, P\left(T_{x / \epsilon} \omega, M_{h} D u\right)\right), D u_{\epsilon}\right) d x d \mu(\omega) \rightarrow \int_{Q}\left(\mathbf{a}^{*}\left(M_{h} D u\right), D u\right) d x
$$

as $\epsilon \rightarrow 0$.

Proof.

$$
\begin{aligned}
& \int_{\Omega} \int_{Q}\left(\mathbf{a}\left(T_{x / \epsilon} \omega, P\left(T_{x / \epsilon} \omega, M_{h} D u\right)\right), D u_{\epsilon}\right) d x d \mu(\omega) \\
& =\sum_{i} \int_{\Omega} \int_{Q_{i}}\left(\mathbf{a}\left(T_{x / \epsilon} \omega, P\left(T_{x / \epsilon} \omega, \xi_{i}\right)\right), D u_{\epsilon}\right) d x d \mu(\omega) .
\end{aligned}
$$

Note that $D u_{\epsilon}$ is bounded in $L^{p}(Q)$ for a.e. $\omega$. Next we show that $\mathbf{a}\left(T_{x / \epsilon} \omega, P\left(T_{x / \epsilon} \omega, \xi_{i}\right)\right)$ is bounded in $L^{r}\left(Q_{i}\right)$, where $r>q$. Since $-\operatorname{div}\left(\mathbf{a}\left(T_{x / \epsilon} \omega, P\left(T_{x / \epsilon} \omega, \xi_{i}\right)\right)\right)=0$ in $3 \times Q_{i}$ (where $3 \times Q_{i}$ is a domain that contains $Q_{i}$ and is surrounded with a ring of size $Q_{i}$ ), using Meyers's theorem (see [13]) we can conclude that

$$
\left\|P\left(T_{x / \epsilon} \omega, \xi_{i}\right)\right\|_{p+\eta\left(Q_{i}\right)} \leq C\left\|P\left(T_{x / \epsilon} \omega, \xi_{i}\right)\right\|_{p\left(3 \times Q_{i}\right)},
$$

where $C$ is independent of $\omega$ and depends only on operator constants and $Q_{i}$. Since $\left\|P\left(T_{x / \epsilon} \omega, \xi_{i}\right)\right\|_{p\left(3 \times Q_{i}\right)}$ is bounded for a.e. $\omega$ (see Lemma 4.2), $\left\|P\left(T_{x / \epsilon} \omega, \xi_{i}\right)\right\|_{p+\eta\left(Q_{i}\right)}$ is also bounded for a.e. $\omega$. From here using $(5)$ for $\mathbf{a}\left(T_{y} \omega, \xi\right)$ we can easily obtain that $\mathbf{a}\left(T_{x / \epsilon} \omega, P\left(T_{x / \epsilon} \omega, \xi_{i}\right)\right)$ is bounded in $L^{r}\left(Q_{i}\right)$, where $r>q$ for a.e. $\omega$. Since $P$ is in $L^{p+\eta}\left(Q_{i}\right)$ for a.e. $\omega$, we can pick $r=q+\eta /(p-1)$. Consequently, $\left(\mathbf{a}\left(T_{x / \epsilon} \omega, \xi_{i}+\right.\right.$ $\left.\left.w_{\xi_{i}}\left(T_{x / \epsilon} \omega\right)\right), D u_{\epsilon}\right)$ is bounded in $L^{\sigma}\left(Q_{i}\right), \sigma>1$. Thus it contains a subsequence that weak* converges to $g_{i}$ for any $i$ and a.e. $\omega$. Since $-\operatorname{div}\left(\mathbf{a}\left(T_{x / \epsilon} \omega, P\left(T_{x / \epsilon} \omega, \xi_{i}\right)\right)\right)=$ 0 in $Q_{i}$, using compensated compactness argument we can obtain that as $\epsilon \rightarrow 0$ 
$g_{i}=\left(\mathbf{a}^{*}\left(\xi_{i}\right), D u\right)$. The latter is because $D u_{\epsilon}$ weakly converges to $D u$ for a.e. $\omega$ and $\mathbf{a}\left(T_{x / \epsilon} \omega, P\left(T_{x / \epsilon} \omega, \xi_{i}\right)\right)$ converges to $\mathbf{a}^{*}(\xi)$ as $\epsilon \rightarrow 0$ (the whole sequence). The weak convergence of $\mathbf{a}\left(T_{x / \epsilon} \omega, P\left(T_{x / \epsilon} \omega, \xi_{i}\right)\right)$ is a consequence of the Birkhoff ergodic theorem. Consequently, as $\epsilon \rightarrow 0$,

$$
\sum_{i} \int_{\Omega} \int_{Q_{i}}\left(\mathbf{a}\left(T_{x / \epsilon} \omega, P\left(T_{x / \epsilon} \omega, \xi_{i}\right)\right), D u_{\epsilon}\right) d x d \mu(\omega) \rightarrow \sum_{i} \int_{\Omega} \int_{Q_{i}}\left(\mathbf{a}^{*}\left(\xi_{i}\right), D u\right) d x d \mu(\omega) .
$$

Thus,

$$
\begin{gathered}
\int_{\Omega} \int_{Q}\left(\mathbf{a}\left(T_{x / \epsilon} \omega, P\left(T_{x / \epsilon} \omega, M_{h} D u\right)\right), D u_{\epsilon}\right) d x d \mu(\omega) \\
\rightarrow \int_{\Omega} \int_{Q}\left(\mathbf{a}^{*}\left(M_{h} D u\right), D u\right) d x d \mu(\omega)
\end{gathered}
$$

For the third term of the r.h.s. of (34) we have the following.

LEMMA 4.6 .

$$
\int_{\Omega} \int_{Q}\left(\mathbf{a}\left(T_{x / \epsilon} \omega, D u_{\epsilon}\right), P\left(T_{x / \epsilon} \omega, M_{h} D u\right)\right) d x d \mu(\omega) \rightarrow \int_{Q}\left(\mathbf{a}^{*}(D u), M_{h} D u\right) d x
$$

as $\epsilon \rightarrow 0$.

Proof.

$$
\begin{aligned}
& \int_{\Omega} \int_{Q}\left(\mathbf{a}\left(T_{x / \epsilon} \omega, D u_{\epsilon}\right), P\left(T_{x / \epsilon} \omega, M_{h} D u\right)\right) d x d \mu(\omega) \\
& =\sum_{i} \int_{\Omega} \int_{Q_{i}}\left(\mathbf{a}\left(T_{x / \epsilon} \omega, D u_{\epsilon}\right), P\left(T_{x / \epsilon} \omega, \xi_{i}\right)\right) d x d \mu(\omega) .
\end{aligned}
$$

Since $\mathbf{a}\left(T_{x / \epsilon} \omega, D u_{\epsilon}\right)$ weakly converges to $\mathbf{a}^{*}(D u)$ in $L^{q}(Q)$ for a.e. $\omega$, and $P\left(T_{x / \epsilon} \omega, \xi_{i}\right)$ converges to $\xi_{i}$ in $L^{p}(Q)$ and is bounded in $L^{p+\eta}(Q)$, similar to the analysis of the second term we can obtain that

$$
\begin{gathered}
\sum_{i} \int_{\Omega} \int_{Q_{i}}\left(\mathbf{a}\left(T_{x / \epsilon} \omega, D u_{\epsilon}\right), P\left(T_{x / \epsilon} \omega, \xi_{i}\right)\right) d x d \mu(\omega) \rightarrow \sum_{i} \int_{Q_{i}}\left(\mathbf{a}^{*}(D u), \xi_{i}\right) d x \\
=\int_{Q}\left(\mathbf{a}^{*}(D u), M_{h} D u\right) d x .
\end{gathered}
$$

For the fourth term of the r.h.s. of (34) we have the following.

LEMMA 4.7.

$$
\int_{\Omega} \int_{Q}\left(\mathbf{a}\left(T_{x / \epsilon} \omega, D u_{\epsilon}\right), D u_{\epsilon}\right) d x d \mu(\omega) \rightarrow \int_{Q}\left(\mathbf{a}^{*}(D u), D u\right) d x
$$

as $\epsilon \rightarrow 0$.

Proof.

$$
\begin{array}{r}
\int_{\Omega} \int_{Q}\left(\mathbf{a}\left(T_{x / \epsilon} \omega, D u_{\epsilon}\right), D u_{\epsilon}\right) d x d \mu(\omega) \\
=-\int_{\Omega} \int_{Q}\left(\operatorname{div}\left(\mathbf{a}\left(T_{x / \epsilon} \omega, D u_{\epsilon}\right)\right), u_{\epsilon}\right) d x d \mu(\omega)=\int_{\Omega} \int_{Q} f u_{\epsilon} d x d \mu(\omega) \\
\rightarrow \int_{Q} f u d x=\int_{Q}\left(\mathbf{a}^{*}(D u), D u\right) d x .
\end{array}
$$


Combining all the estimates we have

$$
\begin{array}{r}
\lim _{\epsilon \rightarrow 0} \int_{\Omega} \int_{Q}\left|P\left(T_{x / \epsilon} \omega, M_{h} D u\right)-D u_{\epsilon}\right|^{p} d x d \mu(\omega) \\
\leq C\left(\int_{Q}\left(\mathbf{a}^{*}\left(M_{h} D u\right), M_{h} D u\right) d x-\int_{Q}\left(\mathbf{a}^{*}\left(M_{h} D u\right), D u\right) d x-\right. \\
-\int_{Q}\left(\mathbf{a}^{*}(D u), M_{h} D u\right) d x \\
\left.+\int_{Q}\left(\mathbf{a}^{*}(D u), D u\right) d x\right) .
\end{array}
$$

This completes the proof of the Theorem 4.3.

Next, it is not difficult to show that the r.h.s. of (42) approaches zero as $h \rightarrow 0$. For this reason we write the r.h.s. as

$$
\begin{array}{r}
\int_{Q}\left(\mathbf{a}^{*}\left(M_{h} D u\right), M_{h} D u\right) d x-\int_{Q}\left(\mathbf{a}^{*}\left(M_{h} D u\right), D u\right) d x-\int_{Q}\left(\mathbf{a}^{*}(D u), M_{h} D u\right) d x \\
+\int_{Q}\left(\mathbf{a}^{*}(D u), D u\right) d x=\int_{Q}\left(\mathbf{a}^{*}(D u)-\mathbf{a}^{*}\left(M_{h} D u\right), D u-M_{h} D u\right) d x .
\end{array}
$$

Using the estimate (25) for $\mathbf{a}^{*}$ and the fact that $\left\|M_{h} D u-D u\right\|_{p} \rightarrow 0$ as $h \rightarrow 0$ we obtain that the r.h.s. of (43) converges to zero as $h \rightarrow 0$. This completes the proof of Theorem 4.1.

Theorem 4.1 shows a way to compute approximation for the gradient of $u_{\epsilon}$, though it involves the solution of the auxiliary problem. Next, we present a computational approach for calculating the approximation for the gradient of the solution using Theorem 4.1. In each element triangular $K$ we solve

$$
\begin{array}{r}
-\operatorname{div}\left(\mathbf{a}_{\epsilon}\left(T_{x / \epsilon} \omega, D v_{\epsilon}\right)\right)=0 \text { in } K, \\
v_{\epsilon}=u_{h} \text { on } \partial K,
\end{array}
$$

where $u_{h} \in S^{h}$ is computed using the numerical homogenization procedure presented in the previous section. Note that $D u_{h} \rightarrow D u$ as $h \rightarrow 0$ in $L^{p}(Q)$, and this allows us to prove the following theorem.

THEOREM 4.8 .

$$
\lim _{h \rightarrow 0} \lim _{\epsilon \rightarrow 0} \int_{\Omega} \int_{Q}\left|D v_{\epsilon}-D u_{\epsilon}\right|^{p} d x d \mu(\omega)=0,
$$

where $v_{\epsilon}$ and $u_{\epsilon}$ are defined by (44) and (4), respectively.

Proof. Because of Theorem 4.1 we just need to show

$$
\lim _{h \rightarrow 0} \lim _{\epsilon \rightarrow 0} \int_{\Omega} \int_{Q}\left|D v_{\epsilon}-P\left(T_{x / \epsilon} \omega, M_{h} D u\right)\right|^{p} d x d \mu(\omega)=0 .
$$

Further, using Theorem 4.3 we obtain that

$$
\lim _{h \rightarrow 0} \lim _{\epsilon \rightarrow 0} \int_{\Omega} \int_{Q}\left|D v_{\epsilon}-P\left(T_{x / \epsilon} \omega, D u_{h}\right)\right|^{p} d x d \mu(\omega)=0 .
$$

Indeed, the homogenized equation for $(44)$ is $-\operatorname{div}\left(\mathbf{a}^{*}(D v)\right)=0$ in $K, v=u_{h}$ on $\partial K$. Thus $v=u_{h}$ in $K$, since $u_{h}$ is a linear function, and $M_{h} D v=D u_{h}$. Thus it remains to show that

$$
\lim _{h \rightarrow 0} \lim _{\epsilon \rightarrow 0} \int_{\Omega} \int_{Q}\left|P\left(T_{x / \epsilon} \omega, D u_{h}\right)-P\left(T_{x / \epsilon} \omega, M_{h} D u\right)\right|^{p} d x d \mu(\omega)=0 .
$$


To show this we note that

(47)

$$
\begin{aligned}
& \int_{\Omega}|P(\omega, \xi)-P(\omega, \zeta)|^{p} d \mu(\omega) \\
& \leq C \int_{\Omega}(\mathbf{a}(\omega, P(\omega, \xi))-\mathbf{a}(\omega, P(\omega, \zeta)), P(\omega, \xi)-P(\omega, \zeta)) d \mu(\omega) \\
& =C \int_{\Omega}(\mathbf{a}(\omega, P(\omega, \xi))-\mathbf{a}(\omega, P(\omega, \zeta)), \xi-\zeta) d \mu(\omega) \\
& \leq C \int_{\Omega}(1+|P(\omega, \xi)|+P(\omega, \zeta) \mid)^{p-1-\alpha}|P(\omega, \xi)-P(\omega, \zeta)|^{\alpha} \times|\xi-\zeta| d \mu(\omega) \\
& \leq C\left(\int_{\Omega}\left(1+|P(\omega, \xi)|^{p}+\left.P(\omega, \zeta)\right|^{p}\right) d \mu(\omega)\right)^{(p-\alpha-1) / p}\left(\int_{\Omega}|P(\omega, \xi)-P(\omega, \zeta)|^{p}\right)^{\alpha / p}|\xi-\zeta|
\end{aligned}
$$

From here using Lemma 4.2 we have

$$
\int_{\Omega}|P(\omega, \xi)-P(\omega, \zeta)|^{p} d \mu(\omega) \leq C\left(1+|\xi|^{p}+|\zeta|^{p}\right)^{(p-\alpha-1) /(p-\alpha)}|\xi-\zeta|^{p /(p-\alpha)} .
$$

Next, denote by $\xi_{K}=D u_{h}$ and $\zeta_{K}=M_{h} D u$ in each $K$. Then for (46) we have

$$
\begin{aligned}
\lim _{h \rightarrow 0} \lim _{\epsilon \rightarrow 0} \int_{\Omega} \int_{Q}\left|P\left(T_{x / \epsilon} \omega, D u_{h}\right)-P\left(T_{x / \epsilon} \omega, M_{h} D u\right)\right|^{p} d x d \mu(\omega) \\
\leq C \lim _{h \rightarrow 0} \lim _{\epsilon \rightarrow 0} \sum_{K} \int_{\Omega} \int_{K}\left(\mathbf{a}\left(T_{x / \epsilon} \omega, P\left(T_{x / \epsilon} \omega, \xi_{K}\right)\right)\right. \\
\left.-\mathbf{a}\left(T_{x / \epsilon} \omega, P\left(T_{x / \epsilon} \omega, \zeta_{K}\right)\right), P\left(T_{x / \epsilon} \omega, \xi_{K}\right)-P\left(T_{x / \epsilon} \omega, \zeta_{K}\right)\right) d x d \mu(\omega) \\
\left.\left.=C \lim _{h \rightarrow 0} \sum_{K} \int_{K}\left(\mathbf{a}^{*}\left(\xi_{K}\right)\right)-\mathbf{a}^{*}\left(\zeta_{K}\right)\right), \xi_{K}-\zeta_{K}\right) d x \\
=C \lim _{h \rightarrow 0} \int_{Q}\left(\mathbf{a}^{*}\left(D u_{h}\right)-\mathbf{a}^{*}\left(M_{h} D u\right), D u_{h}-M_{h} D u\right) d x=0 .
\end{aligned}
$$

In the last step we have used the fact that $D u_{h}-M_{h} D u \rightarrow 0$ in $L^{p}(Q)$ as $h \rightarrow 0$ because of Theorem 3.1 and the fact that $M_{h} D u \rightarrow D u$ in $L^{p}(Q)$ as $h \rightarrow 0$. When taking the limit as $\epsilon \rightarrow 0$ we followed the proof of Lemma 4.4.

Remark. Theorem 4.8 shows that if NMsFEM is employed, then the solution computed in $V_{h}^{\epsilon}$ will approximate the solution of the original equation, (4), in $W_{0}^{1, p}(Q)$ (and in the mean with respect to $\omega$ ).

Remark. The convergence results of section 4 can be generalized to the case for a.e. $\omega$ using essentially the same proof.

5. Concluding remark. In conclusion we would like to note that the numerical homogenization procedure can be applied to more general nonlinear equations (quasimonotone and type $S_{+}($see $[19$, p. 7$])$

$$
-\operatorname{div}\left(\mathbf{a}_{\epsilon}\left(x, u_{\epsilon}, D u_{\epsilon}\right)\right)+a_{0, \epsilon}\left(x, u_{\epsilon}, D u_{\epsilon}\right)=f .
$$

The numerical homogenization will be given as follows:

$$
\left(A^{h, \epsilon} u_{h, \epsilon}, v_{h}\right)=\int_{Q} f v_{h} d x \forall v_{h} \in S^{h},
$$


where

$$
\left(A^{h, \epsilon} u_{h, \epsilon}, v_{h}\right)=\sum_{K} \int_{K}\left[\left(\mathbf{a}_{\epsilon}\left(x, \eta^{u_{h}}, D v_{\epsilon}\right), D v_{h}\right)+a_{0, \epsilon}\left(x, \eta^{u_{h}}, D v_{\epsilon}\right) v_{h}\right] d x
$$

Here $v_{\epsilon}$ is a unique solution of

$$
-\operatorname{div} \mathbf{a}_{\epsilon}\left(x, \eta^{u_{h}}, D v_{\epsilon}\right)=0 \text { in } K
$$

with $v_{\epsilon}=u_{h}$ on $\partial K$, and $\eta^{u_{h}}$ is a constant in $K$ that is equal to the average of $u_{h}$,

$$
\eta^{u_{h}}=\frac{1}{|K|} \int_{K} u_{h} d x
$$

The convergence results for this scheme will be presented elsewhere.

\section{REFERENCES}

[1] G. Allaire, Two-scale convergence: A new method in periodic homogenization, in Nonlinear Partial Differential Equations and Their Applications, Collège de France Seminar, Vol. XII (Paris, 1991-1993), Pitman Res. Notes Math. Ser. 302, Longman Sci. Tech., Harlow, 1994, pp. $1-14$.

[2] T. ARBogast, Implementation of a locally conservative numerical subgrid upscaling scheme for two-phase Darcy flow, Comput. Geosci., 6 (2002), pp. 453-481.

[3] V. Chiadò Piat, G. Dal Maso, and A. Defranceschi, G-convergence of monotone operators, Ann. Inst. H. Poincaré Anal. Non Linéaire, 7 (1990), pp. 123-160.

[4] G. Dal Maso and A. Defranceschi, Correctors for the homogenization of monotone operators, Differential Integral Equations, 3 (1990), pp. 1151-1166.

[5] L. J. Durlofsky, Numerical calculation of equivalent grid block permeability tensors for heterogeneous porous media, Water Resour. Res., 27 (1991), pp. 699-708.

[6] W. E And E. Engquist, The heterogeneous multi-scale methods, Comm. Math. Sci., 1 (2003), pp. 87-132.

[7] Y. R. Efendiev, T. Y. Hou, AND X.-H. Wu, Convergence of a nonconforming multiscale finite element method, SIAM J. Numer. Anal., 37 (2000), pp. 888-910.

[8] Y. R. EFENDIEV AND X. H. WU, Multiscale finite element method for problems with highly oscillatory coefficients, Numer. Math., 90 (2002), pp. 459-486.

[9] T. Y. Hou AND X. H. Wu, A multiscale finite element method for elliptic problems in composite materials and porous media, J. Comput. Phys., 134 (1997), pp. 169-189.

[10] T. Y. Hou, X. H. Wu, AND Z. CAI, Convergence of a multiscale finite element method for elliptic problems with rapidly oscillating coefficients, Math. Comp., 68 (1999), pp. 913-943.

[11] V. V. Jikov, S. M. Kozlov, and O. A. Oleinik, Homogenization of Differential Operators and Integral Functionals, Springer-Verlag, New York, 1994.

[12] O. A. Ladyzhenskaya And N. N. Uraltseva, Linear and Quasilinear Elliptic Equations, Academic Press, New York, 1968.

[13] N. G. Meyers AND A. ElCRAT, Some results on regularity for solutions of non-linear elliptic systems and quasi-regular functions, Duke Math. J., 42 (1975), pp. 121-136.

[14] J. NEČAS, Introduction to the Theory of Nonlinear Elliptic Equations, John Wiley and Sons, Chichester, 1986.

[15] A. PAnkov, Strong G-convergence of nonlinear elliptic operators and homogenization, in Constantin Caratheodory: An Internationial Tribute, World Academic, Teaneck, NJ, 1991, pp. 1075-1099.

[16] A. Pankov, G-Convergence and Homogenization of Nonlinear Partial Differential Operators, Kluwer Academic Publishers, Dordrecht, The Netherlands, 1997.

[17] G. Papanicolaou and S. R. S. Varadhan, Boundary value problems with rapid oscillating random coefficients, Seria Colloquia Mathematica Societatis Janos Bolyai, 27 (1981), pp. 835-873.

[18] U. E. RAĬTUM, On G-convergence of quasilinear elliptic operators with unbounded coefficients, Dokl. Akad. Nauk SSSR, 261 (1981), pp. 30-34.

[19] I. V. Skrypnik, Methods for Analysis of Nonlinear Elliptic Boundary Value Problems, Trans. Math. Monogr. 139, AMS, Providence, RI, 1994. 
[20] M. M. Vainberg, Variational Methods for the Study of Nonlinear Operators, Holden-Day, San Francisco, 1964.

[21] X. H. Wu, Y. R. Efendiev, And T. Y. Hou, Analysis of upscaling absolute permeability, Discrete Contin. Dyn. Syst., Ser. B, 2 (2002), pp. 185-204.

[22] A. ŽAanen, An Introduction to the Theory of Integration, North-Holland, Amsterdam, 1961. 